PROCEEDINGS OF THE

AMERICAN MATHEMATICAL SOCIETY

Volume 133, Number 3, Pages 759-762

S 0002-9939(04)07609-9

Article electronically published on October 7, 2004

\title{
NON-NORMAL DERIVATION AND ORTHOGONALITY
}

\author{
SALAH MECHERI
}

(Communicated by Joseph A. Ball)

\begin{abstract}
The main purpose of this note is to characterize the operators $T \in B(H)$ which are orthogonal (in the sense of James) to the range of a generalized derivation for non-normal operators $A, B \in B(H)$.
\end{abstract}

\section{INTRODUCTION}

Let $B(H)$ be the algebra of all bounded linear operators on an infinite dimensional complex and separable Hilbert space $H$. For $A, B \in B(H)$, let $\delta_{A, B}$ denote the operator on $B(H)$ defined by $\delta_{A B}(X)=A X-X B$. If $A=B, \delta_{A}$ is called the inner derivation induced by $A \in B(H)$. Let

$$
B(H) \supset K(H) \supset C_{p} \supset F(H)(0<p<\infty)
$$

denote, respectively, the class of all bounded linear operators, the class of compact operators, the Schatten $p$-class, and the class of finite rank operators on $H$. All operators herein are assumed to be linear and bounded. Let $\|\cdot\|_{p},\|\cdot\|_{\infty}$ denote, respectively, the $C_{p}$-norm and the $K(H)$-norm. Let $\mathcal{I}$ be a proper bilateral ideal of $B(H)$. It is well known that if $\mathcal{I} \neq\{0\}$, then $K(H) \supset \mathcal{I} \supset F(H)$.

In [1, Theorem 1.7], J.Anderson shows that if $A$ is normal and commutes with $T$, then for all $X \in B(H)$,

$$
\left\|T+\delta_{A}(X)\right\| \geq\|T\| .
$$

Over the years, Anderson's result has been generalized in various ways. Some results concern elementary operators on $B(H)$ such as $X \rightarrow A X B-X$ or $\delta_{A, B}(X)=$ $A X-X B$; since these are not normal derivations, some extra condition is needed in each case to obtain the orthogonality result. In [2], P.B.Duggal established the orthogonality result for $\Delta_{A B}=A X B-X$ under the hypothesis that $(A, B)$ satisfies a generalized Putnam-Fuglede property (which is one way to generalize normality).

Another way to generalize Anderson's result is to consider the restriction of an elementary operator (e.g., $X \rightarrow A X B-X$ ) or $\delta_{A, B}(X)=A X-X B$ to a norm ideal $\left(\mathcal{I},\|\cdot\|_{\mathcal{I}}\right)$ of $B(H)$. Among the results in this direction, Duggal [2] has obtained the orthogonality result for $\left.\Delta_{A B}\right|_{C_{p}}$ (the restriction to the schatten $p$-class $\left.C_{p}\right)$ under the Putnam-Fuglede hypothesis on $(A, B)$, and F.Kittaneh [4]

Received by the editors February 11, 2003 and, in revised form, October 16, 2003.

2000 Mathematics Subject Classification. Primary 47B47, 47A30, 47B20; Secondary 47B10.

Key words and phrases. Derivation, generalized derivation, orthogonality.

This work was supported by the Research Center Project No. Math/1422/10.

(C)2004 American Mathematical Society 
proved the orthogonality result for restricted generalized derivations $\left.\delta_{A, B}\right|_{\mathcal{I}}$ (with the Putnam-Fuglede condition for $(A, B))$.

In this paper we initiate a different approach to generalize Anderson's theorem, one which does not rely on normality via the Putnam-Fuglede condition.

Let $E$ be a complex Banach space. We first define orthogonality in $E$. We say that $b \in E$ is orthogonal to $a \in E$ if for all complex $\lambda$ it holds that

$$
\|a+\lambda b\| \geq\|a\| .
$$

This definition has a natural geometric interpretation, namely, $b \perp a$ if and only if the complex line $\{a+\lambda b \mid \lambda \in C\}$ is disjoint from the open ball $K(0,\|a\|)$, i.e., if and only if this complex line is a tangent one.

Note that if $b$ is orthogonal to $a$, then $a$ need not be orthogonal to $b$. If $E$ is a Hilbert space, then from (1.1) it follows that $\langle a, b\rangle=0$, i.e., orthogonality in the usual sense.

\section{MAIN RESULTS}

Let $\mathcal{A}$ denote a complex Banach Algebra with identity $e$ and let $\sigma_{r}(A), \sigma_{l}(A)$ denote, respectively, the right spectrum and the left spectrum of $A$.

Recall that

$$
a^{n} x-x b^{n}=\sum_{i=0}^{n-1} a^{n-i-1}(a x-x b) b^{i}
$$

for all $x \in \mathcal{A}$.

It easy to see that if $a c=c b$, then for all $x \in \mathcal{A}$ we have

$$
n c b^{n-1}=a^{n} x-x b^{n}-\sum_{i=0}^{n-1} a^{n-i-1}(a x-x b-c) b^{i}
$$

for all $x \in \mathcal{A}$.

Theorem 2.1. Let a be elements of $\mathcal{A},\left(k_{n}\right)$ an increasing sequence of positive integers and $a^{k_{n}}$ converge to $y \in \mathcal{A}$, with $0 \notin \sigma_{r}(y) \cap \sigma_{l}(y)$. If there exists a constant $\alpha$ such that $\left\|a^{n}\right\| \leq \alpha$ for all integers $n$ and if $b$ is the left or the right inverse of $y$, then

$$
\alpha^{2}\|b\|\|a x-x a-c\| \geq\|c\|
$$

for all $x \in \mathcal{A}$ and for all $c \in \operatorname{ker} \delta_{a}$.

Proof. If $x \in \mathcal{A}$, we can write

$$
\left(k_{n}+1\right) c a^{k_{n}}=a^{k_{n}+1} x-x a^{k_{n}+1}-\sum_{i=0}^{k_{n}} a^{k_{n}-i}(a x-x a-c) a^{i} .
$$

It follows that

$$
\left\|c a^{k_{n}}\right\| \leq \frac{2 \alpha}{k_{n}+1}\|x\|+\alpha^{2}\|a x-x a-c\| .
$$

Letting $n$ tend to infinity we get

$$
\|c y\| \leq \alpha^{2}\|a x-x a-c\| .
$$

Now, if $b$ is the right or the left inverse of $y$, we get

$$
\|c\| \leq\|b\| \cdot \alpha^{2}\|a x-x a-c\| .
$$


Corollary 2.1. Let $A \in B(H)$ and assume that there is a constant $\alpha$ such that $\left\|A^{n}\right\| \leq \alpha$ for all integers $n$ and let $\left(k_{n}\right)$ be an increasing sequence of positive integers.

(i) If $A^{k_{n}} \rightarrow C$, with $0 \notin \sigma_{r}(C) \cap \sigma_{l}(C)$, then

$$
\alpha^{2}\|A X-X A-T\| \geq\|T\|,
$$

for all $X \in B(H)$ and for all $T \in \operatorname{ker} \delta_{A}$.

(ii) If $A^{k_{n}} \rightarrow C+K$, with $K$ compact and $0 \notin \sigma_{r}(C) \cap \sigma_{l}(C)$, then

$$
\alpha^{2}\|A X-X A-T-K\| \geq\|T\|
$$

for all $X \in B(H)$ and for all $T \in \operatorname{ker} \delta_{A}$.

Proof. It is a simple consequence of the above theorem.

Theorem 2.2. Let $A \in B(H)$ satisfying $A^{m}=I$ for some integer $m$. Then

$$
\alpha^{2}\|A X-X A-T\| \geq\|T\|,
$$

for all $X \in B(H)$ and for all $T \in \operatorname{ker} \delta_{A}$.

Proof. Since $A^{n} \in\left\{I, A, A^{2}, \ldots, A^{m-1}\right\}$ for all integers $n,\left\|A^{n}\right\| \leq \alpha, n \in N$ and $A^{k_{n}}=I$, where $k_{n}=n m, n \in N$. It suffices to apply Corollary 2.1 (i).

Corollary 2.2. Let $A, B \in B(H)$ satisfying $A^{m}=I$ and $B^{m}=I$ for some integer $m$. Then

$$
\|A X-X B-T\| \geq\|T\|,
$$

for all $X \in B(H)$ and for all $T \in \operatorname{ker} \delta_{A, B}$.

Proof. It is sufficient to take on $H \oplus H$,

$$
N=\left(\begin{array}{ll}
A & 0 \\
0 & B
\end{array}\right), Y=\left(\begin{array}{cc}
0 & X \\
0 & 0
\end{array}\right), S=\left(\begin{array}{ll}
0 & T \\
0 & 0
\end{array}\right) .
$$

It is clear that $N^{m}=I, N S=S N$, i.e., $S \in \operatorname{ker} \delta_{N}$ and

$$
N Y-Y N=\left(\begin{array}{ll}
0 & A X-X B \\
0 & 0
\end{array}\right) .
$$

By applying the above corollary it follows that

$$
\|A X-X B-T\|=\|N Y-Y N\| \geq\|S\|=\|T\| .
$$

Remark 2.1. Note that the operators mentioned above are not in general normal or isometric. To see that, it is enough to consider on 2-dimensional $H$ the operator

$$
A=\left[\begin{array}{ll}
1 & \alpha \\
0 & -1
\end{array}\right],
$$

where $\alpha \in C \backslash\{0\}$. For this $A^{2}=I$ and $A$ is not normal or isometric.

About the operators $A^{m}=I$. Such operators are similar to unitary operators. (Indeed, $A^{m}$ is a contraction implies $A$ is similar to a contraction $C$ implies $C^{m}=I$ implies $C$ is unitary.) One can show: If $\phi$ is a power bounded operator (on a Banach algebra $\mathcal{A}$, to itself), $S=I-\phi$ and $T=\phi-(\phi)^{2}$, then $S^{-1}(0) \perp_{\alpha} T(A)$. If the algebra $\mathcal{A}$ is $B(H)$ and $a$ is polynomially bounded, then there exists a quasi-affinity $X$ and a contraction $C$ (on some Hilbert space) such that $X C=a X$. 
A simple calculation shows that

$$
a^{n} x b^{n}-x=\sum_{i=0}^{n-1} a^{n-i-1}(a x b-x) b^{n-i-1}
$$

for all $x \in \mathcal{A}$.

It is easy to see that if $a c b=c$, then for all $x \in \mathcal{A}$ we have

$$
-n c=a^{n} x b^{n}-x-\sum_{i=0}^{n-1} a^{n-i-1}(a x b-c) b^{n-i-1} .
$$

Thus, if $\left\|a^{n}\right\| \leq 1$ and $\left\|b^{n}\right\| \leq 1$, then

$$
\|a x b-x-c\| \geq\|c\|,
$$

for all $x \in \mathcal{A}$ and for all $c \in \operatorname{ker} \Delta_{a b}$. We also have

$$
\|a x-x b-c\| \geq\|c\|,
$$

if $\left\|a^{n}\right\| \leq 1$ and $\left\|b^{n}\right\| \leq 1$.

Note that Theorem 2.1 and its corollary still hold if we consider $a x a-x$ instead of $a x-x a$. Also Theorem 2.2 and its corollary still hold if we consider $a x b-x$ instead of $a x-x b$. Let $\tilde{\delta}_{A}$ be the image of $\delta_{A}$ under the canonical projection of $B(H)$ onto the Calkin algebra $\mathcal{B}$ defined by

$$
\tilde{\delta}_{A}(\tilde{X})=\tilde{A} \tilde{X}-\tilde{X} \tilde{A}
$$

Since in (1) $K$ is an arbitrary compact operator, then

$$
\|\tilde{A} \tilde{X}-\tilde{X} \tilde{A}-\tilde{T}\| \geq\|\tilde{T}\|
$$

\section{ACKNOWLEDGMENT}

I would like to thank Professor B.P.Duggal and the referee for their useful remarks.

\section{REFERENCES}

[1] J. Anderson, On normal derivations, Proc. Amer. Math. Soc. 38-1(1973), 135-140. MR 47:875

[2] B.P. Duggal, A remark on normal derivations, Proc. Amer. Math. Soc. 126-7(1998), 2047-2052. MR98h:47050

[3] F. Kittaneh, Operators that are orthogonal to the range of a derivation, J. Math. Anal. Appl. 203(1996), 863-873. MR97f:47033

[4] F.Kittaneh, Normal derivations in norm ideals, Proc. Amer. Math. Soc., 123-6(1995)1779-1785. MR95g:47054

[5] P.J. Maher, Commutator approximants, Proc. Amer. Math. Soc. 115(1992), 995-1000. MR92j:47059

[6] S. Mecheri, On minimizing $\|S-(A X-X B)\|_{p}$, Serdica Math. J. 26 (2000), no. 2, 119-126. MR2001j:47033

[7] S. Mecheri, On the orthogonality in von Neumann-Shatten classes, Int. Jour. Appl. Math, 8(2002), 441-447. MR2003b:47063

[8] S. Mecheri and A.Bachir, Generalized derivation modulo the ideal of all compact operators, Int.Jour.Math.Math.Sc., 32.8(2002), 501-506. MR2003i:47038

Department of Mathematics, King Saud University College of Science, P.O. Box 2455, RiYadh 11451, SaUdi Arabia

E-mail address: mecherisalah@hotmail.com 